\title{
Development and Inadequacy: Research on the Rights and Interests Protection of the Subjects in Chinese Drug Clinical Trials
}

\author{
Juan Song ${ }^{1, a}$ Shanshan Mei $^{1, b}$ \\ ${ }^{1}$ Xi'an Medical University, Xi'an, Shaanxi, China, 710021 \\ a email, ${ }^{\mathrm{b}}$ email,
}

Keywords: Drug Clinical Trial; Subjects; Drug Clinical Trial Liability Insurance

\begin{abstract}
Chinese drug clinical trial subjects are large, and constantly improve the protection of the legitimate rights and interests of this group is the Chinese government for many years the goal. By continuously improving the legislation, strengthening the supervision and management of the relevant subjects, regulating the operation of a series of measures such as the operation of the ethics committee, the level of the rights and interests of the Chinese drug clinical trial subjects is progressing rapidly. But there is still need to improve the aspects, such as the law of the update is lagging behind, the sponsor and drug clinical trial organization of the process of inadequate regulation, part of the ethics committee is not standardized operation, the insurance link defects affect the realization of the rights of the subjects. It is proposed to better protect the rights and interests of the subjects and promote the progress of medical technology by timely revision of the law, to achieve unified institutional supervision, to ensure the independence of the ethics committee, to strengthen the drug clinical trial liability insurance.
\end{abstract}

\section{Introduction}

Over the past decade, the number of Chinese drug registration increased year by year, drug clinical trials as a drug registration must be frequent links, medical science research to make progress and benefit the community and human. However, drug clinical trial subjects (hereinafter referred to as "subjects") are in a weak position due to factors such as economic, social status, expertise and access to information, and thus have a huge risk of their own health. Chinese current group of participants has reached about 500,000 people, the legitimate rights and interests of this large group should also be the special protection of the law. The Chinese government has been working to improve the level of protection of the rights and interests of subjects' years of effort, made great achievements. This article will discuss the progress and the shortcomings and countermeasures in this field.

\section{China Has Made Significant Progress in Improving the Level of Rights and Interests of Subjects}

The Legal Norms Related to the Protection of the Rights and Interests of the Subjects Are Becoming More and More Perfect. From 2003 onwards, the State Food and Drug Administration (hereinafter referred to as "SCFDA." In 2013 the agency upgraded to the State Food and Drug Administration, China Food and Drug Administration, hereinafter referred to as "CFDA" ) And the Ministry of Health have issued a series of regulations such as Good Clinical Practice (hereinafter referred to as "GCP") to regulate drug clinical trial activities and to request the protection of subjects' rights and interests.

The GCP clarifies that the ethics committee and the informed consent form are the main measures to protect the rights and interests of the subjects and provide specific provisions for the composition, duties, specific scope of examination and working procedures of the ethics committee from the perspective of protecting the rights and interests of the subjects. At the same time, the legislature has also been concerned about the new situation, such as international multicenter drug clinical trials in different countries appear social taboos, religious customs and other differences on 
the protection of the new questions raised by the subjects.

The efforts of Chinese subjects to protect the rights and interests started late, but the legislation focus on reference, learn from international experience. For example, the GCP established the Principles for the Protection of Subjects as set out in the Declaration of Helsinki and annexed the Declaration as an endorsement to the effect of domestic law. The Declaration of the Helsinki Declaration, the Universal Declaration on the Human Genome and Human Rights, the International Ethical guidelines for biomedical research involving human beings, and so on. The basic principles of the protection of the rights and interests of the subjects and the basic rights of the subjects are established in the Chinese legal documents to ensure that Chinese protection of the rights and interests of the subjects can be synchronized with the international.

Strengthen the Supervision of Sponsor, Drug Clinical Trial Institutions and Researchers. In China, sponsors to carry out drug clinical trial activities must be approved by CFDA. If it is a foreign pharmaceutical company, through its branch in China or commissioned by the Chinese agency to apply. CFDA assesses the results of the sponsor's preclinical study through the approval process to avoid the potential risks to the subject.

Medical institutions to carry out drug clinical trial activities of the qualification also need to get CFDA permission and qualification is valid for 3 years. From the official website of the CFDA published on the list of test institutions, access to the qualification of the basic institutions of the three hospitals.

Researchers as a participant in the development of a program to develop and implement a program, is the most direct protection of the subjects of the interests of the party, bear to explain the situation to the subjects, obtain the consent of the subjects, report adverse reactions and timely treatment of the responsibility. The GCP has made a request for its qualifications, clinical experience, and the domination of personnel and equipment.

In addition, the GCP also requires the sponsor to appoint an inspector to supervise the testing process, including violations of the trial program that may occur in the course of the trial, and may be subject to the benefit of the subject; the modification of the test plan is subject to approval by the Ethics Committee; Serious adverse events should be reported to the Ethics Committee in a timely manner.

The Review of the Ethics Committee Is Becoming More Standardized. In China, the Ethics Committee, which is located in the test bodies, is directly responsible for reviewing whether the drug clinical trial activities are in line with ethical requirements. All agency ethics committees are required to file with CFDA. The composition of the ethics committee, the principles of the review, the contents of the review, the review procedures and other aspects should be consistent with the relevant provisions. In order to ensure the comprehensiveness of ethical review, the members of the Ethics Review Committee are required to come from biomedical, management, ethics, law, sociology and the number of people shall not be less than 5, to protect the rights of female subjects, different requirements Gender members. In reviewing the pilot program, it is important to review whether the trial program adequately considers ethical principles, including the risks, benefits and benefits of the study, subjects and others, and the scientific nature of the trial design; (Or its family members, guardians, legal agents) to provide information on the test is complete and easy to understand, access to informed consent method is appropriate; subjects due to participate in clinical trials were damaged or even death, given Treatment and / or insurance measures.

Special Protection Is Given to Special Subjects. Special subjects, due to their mental development, gender, social status and other factors, often in the choice of whether to participate in drug clinical trials activities can not fully express their true will, which may put themselves in a huge trial risk do not know.

Chinese relevant laws have given special attention to this group. It is clear that subjects who lose or lack the ability to defend their rights and interests, including children, pregnant women, mentally handicapped persons, mentally ill persons, prisoners, and those with poor economic conditions and low educational level, should be specially protected. The GCP has provided for the confirmation of the informed consent of the incapacitated person and the child. 


\section{The Deficiencies in the Field of Protection of Chinese Drug Clinical Trials}

Legislation Is Scattered, the Provisions Is Simple and Modify Is Hysteresis. With the expansion of the scale of drug clinical trials and the increase in the level of human rights protection, many countries have developed a domestic law to focus on the rights of subjects, such as the French "human test subjects' rights protection law." Chinese current legal documents related to the protection of subjects are mainly CFDA and SFDA to develop, a few norms is the original Ministry of Health to develop. The situation of decentralized legislation may lead to normative conflicts, such as the access of the ethics committee and the access of the testing agency, and the CFDA and the National Guard Commission's power boundaries are not very clear. This will bring obstacles to the protection of the rights and interests of the subjects.

The existing norms only provide for the special protection of special groups such as children, pregnant women and those with mental retardation. However, there is a lack of further detailed provisions on how to realize the right of informed consent, ethical review and ex post compensation. The GCP only provides for special treatment of children and incapacitated persons in informed consent. Such a situation may lead to the protection of special groups can not be implemented due to lack of maneuverability.

The law stipulates that it is necessary to adjust and perfect according to the change of practice. At present, Chinese domestic legislation is lagging behind. The "Review of Ethical Review of Human Biomedical Research", which came into effect on December 1, 2016, represents Chinese latest legal developments in this field, but there are still other things that are not available for medical institutions drug test institutions have legal effect. In addition, there are very few provisions on the rights and obligations of the Contract Research Organization (CRO) in drug clinical trials, which are present in a large number of drug clinical trials. This situation does not match the actual role of the contract research organization in the course of drug clinical trials. In addition, the 2013 Helsinki Declaration was amended, with multiple modifications involving the protection of the rights and interests of the subjects. GCP and other documents will be the "Helsinki Declaration" as an annex to the Declaration in China has a legal effect. Although the above-mentioned legal documents have already initiated the revision process, the official text has not been published.

Part of the Agency Ethics Committee Operation Is Not Standardized and It Affects the Protection of the Rights and Interests of Subjects. The Institutional Ethics Committee is responsible for ethical review of pilot activities carried out by drug clinical trial bodies and is an important institution for safeguarding the rights and interests of subjects.

First, the ethics committee's independence is not enough. In practice, some members of the composition of the ethics committee do not meet the requirements of the subject, the lack of ethics or law and other social science experts. The ethics committee of the organization is usually set up in the testing organization, and it is necessary to provide the office space, the necessary office expenses and the auxiliary personnel by the testing institution. The ethical examination may take place from the benefit of the test institution and neglect the protection of the subject.

Second, ethical review is not strict. The review of some ethics committees has not been carried out in strict accordance with the relevant provisions. For example, the sponsor does not purchase insurance for the subject will affect the implementation of the trial compensation. But many did not buy insurance test program through the ethical review.

Insurance Links Are Flawed and It Can Not Fully Guarantee the Realization of the Rights of the Subjects. The GCP requires the sponsor to purchase insurance for the subject, with the aim of ensuring that the subject is compensated in a timely manner after the test is damaged. This is an important measure to protect the rights and interests of the subjects, but in the specific implementation will encounter the following questions:

First, there are few insurance companies that develop drug liability for drugs specifically for the subject. The development of the insurance is still the initial stage, the main reason is that the insurance is too professional, the insurance company risk insurance needs of professional medical expertise to assess the risk of insurance drugs, sales staff also need to have the background of professional knowledge And professional communication with customers. Chinese insurance 
industry practitioners have a lower threshold and insurance companies generally can not have the professional insurance product development and design, risk insurance and sales staff. At present, only four insurance companies have developed drug clinical liability insurance, the applicant's selectivity is small.

Second, the ethical review did not take the insured as a condition of adoption. Although the GCP has clearly defined the sponsor's obligation to purchase insurance for the subject, the Ethics Committee's review does not explicitly include the matter as a condition that the sponsor must have. In practice, some sponsors and CROs did not fulfill their insurance obligations and passed the ethical review. In the event of adverse drug reactions in the course of the trial, it is easy for the subject to point to the test institution and the investigator and cause social group events.

Finally, there are loopholes in the regulation of foreign insurance practices. In international multicenter trials, it is possible for sponsors to purchase insurance for Chinese subjects abroad. However, the relevant provisions do not require the sponsor to the patient and drug clinical trial institutions to provide insurance contracts in Chinese translation, nor does the regulation require the authorities to conduct an audit of the applicant when the insurance contract for the record. If the subject can not actively cooperate with the defendant or the court to provide insurance translation of the Chinese translation and actively handle the claims procedures, not only the subject's right to know can not be fully realized, but also will be the subject of the rights and interests of the dispute or into the proceedings, directly affect the degree of achievement of the subject's compensation. In the case of the shock of the Chinese drug court in Chaoyang District People's Court in Beijing in 2013, the Chinese translation of the insurance contract, which refused to provide foreign insurance coverage, led to delays in the case for several years.

\section{Comments and Suggestions}

The legislature should promptly revise the relevant provisions, reflecting the international and domestic development of new developments. Can be regularly revised and the "Helsinki Declaration" and other international treaty amendments and synchronization, and drug clinical trials activities in the emergence of new phenomena and new problems, such as how to limit the "trial drug" frequently participate in experimental activities; how to regulate CRO How to strengthen the supervision of transnational drug testing activities and other issues in a timely manner to make a legal response to enhance the legal norms of the pilot activities of the guiding role.

The concentration of supervision of drug clinical trial activities is exercised by a regulatory authority. According to the setting of the state organs in China, it can be regulated by CFDA. Change the current status of the National Health Commission and CFDA in the ethics committee supervision and drug clinical trial institutions to participate in the field of qualification to jointly exercise the status quo, improve the efficiency of regulation, but also to avoid the government may bring a number of standard conflict.

Continuing to strengthen the supervision of the Ethics Committee, you may attempt to conduct an ethical review by a third party independent of the sponsor and the testing agency. By ensuring the independence of the ethics committee, the objective and impartiality of the ethical review results is enhanced.

You can also try to standard documents through the mandatory requirements of the sponsor to buy drug clinical trial liability insurance (11), while exploring the medical liability insurance and other specialized insurance, decentralized trial risk, to ensure the effective implementation of the trial compensation.

\section{References}

[1] Man Hongjie. Medical human body test special subject protection research [J]. Dong Yue Lun, 2012, 33 (4): 180-185. 
[2] Yu Xiaoyong, Tian Kan. On drug clinical minors in the protection of rights and interests [J]. Chinese Journal of New Drugs, 2014, 23 (15) 1790-1794.

[3] Yao Shusen, Fan Zhen. "Helsinki Declaration" revised and the rights and interests of subjects [J]. Chinese Hospital, 2014, 8 (2): 51-53.

[4] Chen Zhaohui, Yin Mei, etc. Investigation and analysis of the status quo of hospital ethics review committee in Heilongjiang province [J] .Journal of Medicine and Law, 2014,6 (5): 16-19.

[5] Zhang Li, Liu Xiaoyan.On the protection of rights and interests of subjects in clinical trials theoretical basis, practical dilemma and legal approach [J]. Journal of Chongqing University of Technology (Social Science), 29 (12) 97-104.

[6] Shan Ailian, Liang Yan, etc.. In the new drug and medical equipment clinical trial for the subjects to provide insurance investigation and analysis[J]. Chinese Journal of Clinical Pharmacology .2013,29 (7): 483-487.

[7] Wang Chaoqun. Clinical trial insurance status and countermeasures[M]. Guangzhou: Jinan University .2014.

[8] Man Hongjie. On the transnational human test subjects to protect - based on the review of international norms [J]. Journal of Shandong University (Philosophy and Social Sciences Edition) 2012 (4) 39-46.

[9] Zheng Yifei, Zhang Fumin et al. Problems and Countermeasures in the Construction of Hospital Ethics Committee [J]. Chinese Journal of Hospital Administration, 2011, 31 (6): 42-44. 